\title{
Integrative Visual Analysis of the Effects of Alternative Splicing on Protein Domain Interaction Networks
}

\author{
Dorothea Emig ${ }^{1}$, Melissa S. Cline ${ }^{2}$, Karsten Klein ${ }^{3}$, Anne Kunert ${ }^{3}$, Petra Mutzel ${ }^{3}$, Thomas \\ Lengauer $^{1}$ and Mario Albrecht ${ }^{1,4}$
}

\author{
${ }^{1}$ Department of Computational Biology and Applied Algorithmics, Max Planck Institute for \\ Informatics, Campus E1.4, 66123 Saarbrücken, Germany
}
${ }^{2}$ Department of Molecular Cell and Developmental Biology, University of California, Santa Cruz, CA 95064, USA
${ }^{3}$ Department of Computer Science, Dortmund University of Technology, Otto-Hahn-Str. 14, 44227 Dortmund, Germany

\begin{abstract}
Summary
Proteins and their interactions are essential for the functioning of all organisms and for understanding biological processes. Alternative splicing is an important molecular mechanism for increasing the protein diversity in eukaryotic cells. Splicing events that alter the protein structure and the domain composition can be responsible for the regulation of protein interactions and the functional diversity of different tissues. Discovering the occurrence of splicing events and studying protein isoforms have become feasible using Affymetrix Exon Arrays. Therefore, we have developed the versatile Cytoscape plugin DomainGraph that allows for the visual analysis of protein domain interaction networks and their integration with exon expression data. Protein domains affected by alternative splicing are highlighted and splicing patterns can be compared.
\end{abstract}

\section{Introduction}

In recent years, many pairwise protein-protein interactions have been uncovered using experimental high-throughput techniques like yeast-2-hybrid or computational prediction methods [1]. One problem is that proteins found to interact, in fact, may not be contained in the same cellular compartment or the same tissue. Additionally, alternative splicing events complicate the analysis of protein interaction data further. It has been shown that alternative splicing affects up to $70 \%$ of all human genes, resulting in at least two protein isoforms produced by a single gene [2]. However, most protein interaction datasets do not distinguish between different isoforms although alternative splicing can affect the binding regions of proteins, which may alter protein interactions [3].

Several studies have been performed that address the question to what extent alternative splicing affects protein domains or conserved motifs. For example, Resch and colleagues designed a database containing splice variants obtained from human EST data and the first draft of the human genome sequence [4]. They aimed at detecting those Pfam and SMART domains that

\footnotetext{
${ }^{4}$ To whom correspondence should be addressed. E-mail: mario.albrecht@mpi-inf.mpg.de
} 
are preferentially inactivated by alternative splicing events. To this end, they also performed case studies on several domains known to be responsible for mediating protein-protein interactions. Another study to uncover potential functional changes by alternative splicing concentrated on detecting differences of InterPro domains and BLOCKS motifs contained in protein isoforms [5]. This work revealed that about $30 \%$ of the alternatively spliced genes vary in their domain or motif composition.

To monitor the occurrence of alternative splicing events experimentally, Affymetrix has recently released a microarray called Exon Array [6]. The probes on this microarray have been specifically designed for detecting exon expression instead of whole gene expression. Using this microarray, Clark and colleagues performed a study on the detection of protein isoforms [7] and on their assignment to different tissues. This approach resulted in the identification of tissue-specific probesets, which preferentially occur in tissues like cerebellum and testis. Thus, these microarray data may provide insight into the functional differences of protein isoforms and their roles in tissue development.

Furthermore, it is known that disrupting the control of alternative splicing can be the cause of diseases [8]. Alternative splicing events can lead to the inclusion or exclusion of whole exons in the transcript sequence or cause shifts in the reading frames of transcripts, resulting in modified protein sequences and functions. For instance, Gardina and coworkers compared samples of healthy and colon cancer tissues using Affymetrix Exon Arrays and identified a number of differentially expressed genes and novel splicing events that might result in disease-causing protein isoforms [9].

To analyze and visualize the effects of alternative splicing events on protein interactions and their underlying domain interactions, we have developed the new Cytoscape plugin DomainGraph (http://domaingraph.bioinf.mpi-inf.mpg.de/). Cytoscape is a free, open-source Java software platform for the visual analysis of molecular interaction networks [10]. The plugin DomainGraph decomposes a given protein interaction network into the underlying domain-domain interactions [11] and supports the integration of Affymetrix Human Exon Array data. Alternative splicing events observed according to the integrated expression data are highlighted in the network, and the effects on protein domain interactions can easily be illustrated. Therefore, differences in the protein repertoire of tissue samples as well as discrepancies between healthy and diseased tissues can be visually investigated.

\section{Software Description}

\subsection{Domain Graph Definition}

We use undirected graphs to represent the interactions between proteins and their underlying domain interactions [12]. A domain graph is defined as a graph $G=(V, E)$ with a node set $V$ and an edge set E. Proteins and their constituent domains are nodes in the graph, forming the union set $\mathrm{V}$ of protein and domain nodes. There are three kinds of edges, indicating the occurrence of a specific domain in a protein or an interaction between two proteins or two domains. Thus, E consists of protein-protein interaction edges, domain-domain interaction edges, and proteindomain linkers connecting domains to the corresponding proteins. 


\subsection{Domain Graph Generation}

The DomainGraph plugin is fully integrated into the Cytoscape application and can be started via the Plugins menu. In a first step, the user imports a file containing pairwise protein-protein interactions and specifies the species. The plugin supports UniProtKB accession numbers [13] for twelve different species, namely, H. sapiens, M. musculus, R. norvegicus, G. gallus, S. cerevisiae, S. pombe, D. melanogaster, A. thaliana, C. elegans, D. rerio, P. falciparum, and E. coli. For human protein interaction networks, Ensembl transcript and peptide identifiers [14] are also accepted. In the next step, the user has to select one of the eleven available domaindomain interaction datasets containing interactions between Pfam-A domains [15], which are used to compute the domain interactions underlying the imported protein interaction network. DomainGraph provides two structurally derived domain interaction datasets, iPfam [16] and 3did [17], and nine computationally derived datasets: InterDom [18], LLZ [19], DPEA [20], DIMA [21], LDSC [22], RDFF [23], RCDP50 [24], APMM [25], and LP [26]. The last step for the user is to choose one of the three initial views (Section 2.6) for the resulting domain graph.

After submitting the selected data, the domain graph is automatically generated (Section 2.9). Each created domain graph is labeled with the name of the protein data file, the domain-domain interaction method, and the currently selected view. This helps the user to keep track of all generated domain graphs. The nodes are labeled with their names, and the interaction edges are annotated with confidence scores if provided by the user-selected domain interaction dataset. For that purpose, the domain interaction edges are labeled with their corresponding confidence scores in a first step. The protein interaction edges are then annotated with the maximum of the confidence scores provided by the underlying domain interactions. If a user-imported protein

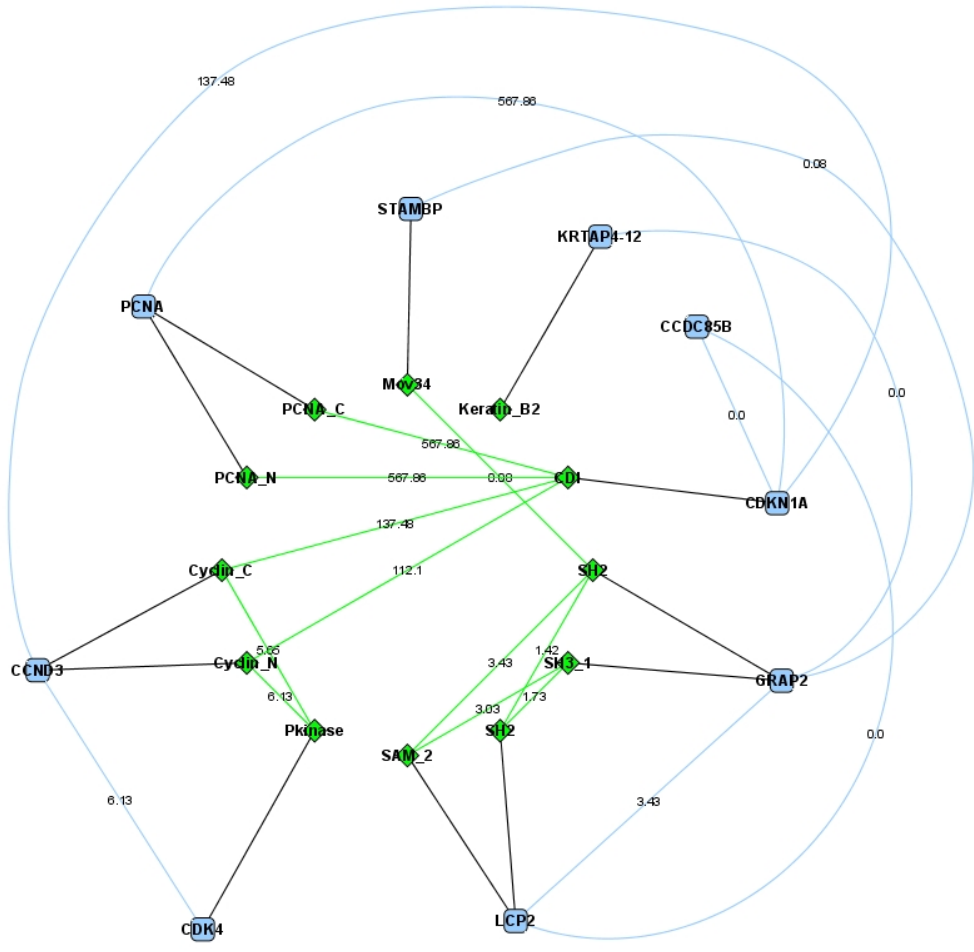

Figure 1: Sample domain graph. Protein nodes (blue rectangles) are located on the outer circle, domain nodes (green diamonds) on the inner circle. Protein interaction edges are shown in blue, domain interaction edges in green, and protein domain linkers in black (see Section 2.4). 
interaction cannot be traced to any underlying domain interaction, the confidence score 0 is assigned to the protein interaction edge, indicating the uncertainty of the protein interaction. Figure 1 shows an example of the DomainGraph plugin output using a small subnetwork of the protein interaction network by Rual et al. [27].

\subsection{Domain Graph Integration with Expression Data}

Once a domain graph has been created, the user has the possibility of integrating own Affymetrix Human Exon 1.0 ST Array data. For this purpose, a pre-processed expression data file and a p-value data file are needed. Both files can easily be generated by applying the "apt-probeset-summarize" method included in the Affymetrix Power Tools (APT) [28]. The expression data file can be directly retrieved from the raw Affymetrix CEL files, for instance, using the provided RMA or PLIER methods for normalization and background subtraction. The processed expression data file then contains a list of probeset ids and their respective expression values. The p-value data file can also be obtained from the CEL files using the DABG method of APT. This method assigns a p-value to each probeset id, which can be used as a presence or absence call for the respective probeset. The default threshold for the presence of a probeset is set to 0.05 but can be modified by the user.

The integration of these data into the domain graph is performed automatically (see Section 2.9 for a detailed description), and the user can directly visualize the occurrence of alternative splicing events in domain-coding regions. This visualization points to alternative splicing events that may disturb certain protein and domain interactions of interest. Moreover, gene expression and suppression is detected, and the user can thus distinguish between the loss of interactions due to alternative splicing and gene suppression events. A sample domain graph with integrated

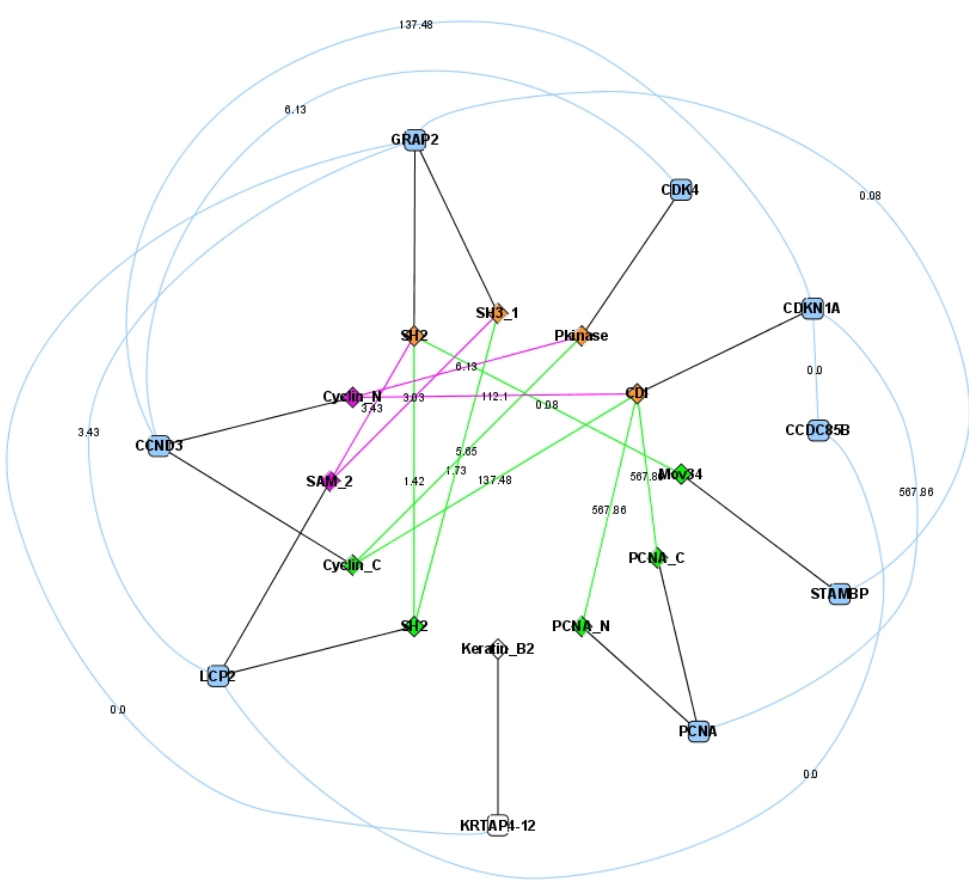

Figure 2: Sample domain graph from Figure 1 with integrated exon expression data. Pink domain nodes indicate the loss of the domain due to alternative splicing events. Orange nodes represent indirectly affected domains. White nodes have no Affymetrix probesets assigned. 
exon expression data from the tissue testis [7] is shown in Figure 2, and a detailed description of the node shapes and node and edge colors is given in Section 2.4.

\subsection{Domain Graph Representation}

The definition of the appearance of nodes and edges, for example, the node shapes and colors, are collectively referred to as the Visual Style of the network within Cytoscape. A Visual Style is dependent on arbitrary node and edge attributes, which can be assigned to the respective node and edge objects. The DomainGraph plugin introduces a predefined Visual Style for the created domain graphs so that the user can easily distinguish, for instance, between protein and domain nodes or protein and domain interaction edges. The different node and edge types introduced by the plugin are described below and their visual appearances in Cytoscape are depicted in Figures 3 and 4.

\subsubsection{Domain Graph without Expression Data Integration}

The plugin provides the following default shapes and colors for the protein and domain nodes and the different types of edges:

- Protein nodes are represented by rounded rectangles, which are colored light blue.

- Domain nodes are shown in a diamond shape and colored green.

- Protein-protein interaction edges are colored light blue, domain-domain interaction edges in green, and edges linking proteins and constituent domains are colored black.

\subsubsection{Domain Graph with Expression Data Integration}

After Affymetrix Human Exon Array data has been integrated into the domain graph, occurrences and effects of alternative splicing events as well as gene suppression events are highlighted. To enable the user to notice such occurrences and their effects on the protein and domain interaction network, the visual style introduced above has been extended. The node and edge shapes are not changed, but the coloring of nodes and edges are adjusted as follows:

- Expressed protein: If the gene producing the respective protein is expressed according to the classification resulting from the interpretation of the Affymetrix data, the protein is regarded expressed and the corresponding node is colored light blue.

- Suppressed protein: If the Affymetrix data indicate that the gene coding for a certain protein is not expressed, the protein is regarded as suppressed and the protein node is colored gray.

- Expressed domain: If the Affymetrix data show that the domain-coding region of the protein is expressed, the corresponding domain node is colored green. 
- Spliced domain: A node is colored pink if it represents a domain that is partly or completely missing due to alternative splicing events.

- Indirectly affected domain: An indirectly affected domain node is a domain that is not subject to alternative splicing but is affected indirectly because it would interact with at least one domain that may be spliced out, which implies the loss of this interaction; an indirectly affected domain node is colored orange.

- Proteins and domains without assigned Affymetrix annotation are colored white.

- Protein/domain combination node: This node type may only occur when two domain graphs are analyzed, more specifically, when the intersection, union or difference of two domain graphs is computed (Section 2.8). If a protein/domain is contained in both analyzed domain graphs, but has different types assigned (e.g., expressed in one graph and suppressed in the other), this is indicated by a dark blue protein/domain node.

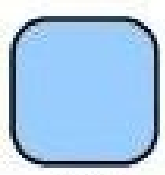

(1)

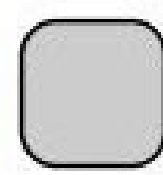

(2)

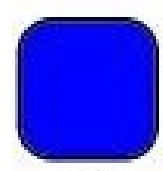

(3)

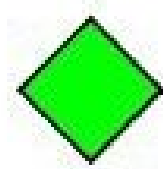

(4)

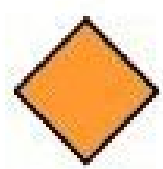

(5)

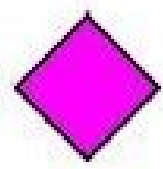

(6)

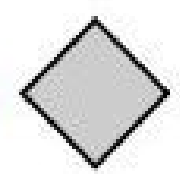

(7)

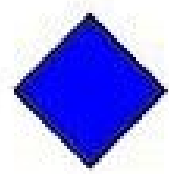

(8)

Figure 3: Node types defined by the DomainGraph Visual Style: (1) normally expressed protein, (2) suppressed protein, (3) protein combination, (4) normally expressed domain, (5) indirectly affected domain, (6) spliced domain, (7) suppressed domain, and (8) domain combination.

(3)

(4)

(5)

(6)

Figure 4: Edge types defined by the DomainGraph Visual Style: (1) occurring protein-protein interaction, (2) protein-domain linker, (3) occurring domain-domain interaction, (4) loss of domaindomain interaction due to alternative splicing, (5) loss of protein/domain interaction due to gene suppression, and (6) protein/domain combination edge.

The edge colors are also further refined to distinguish between different biological types:

- Protein-domain linkers connecting proteins with their constituent domains are colored black.

- A user-defined protein-protein interaction occurring between two expressed protein nodes is supposed to exist and is colored light blue.

- A domain-domain interaction formed by expressed or indirectly affected domains only is regarded as present and is colored green.

- A domain-domain interaction for which at least one of the interacting domains is spliced out is colored pink to indicate the loss of the domain interaction.

- The loss of protein and domain interactions due to gene suppression is shown in gray.

- Protein/domain combination edge: This type may only occur when analyzing two domain graphs. If an edge is present in both domain graphs, but has different edge types annotated, the edge in the resulting domain graph is colored dark blue. 


\subsection{Domain Graph Layout}

The visual style provided by DomainGraph enables the user to readily distinguish between protein and domain nodes and, in case of integrated expression data, also between the different protein and domain types. However, standard layout algorithms like a grid layout or forcedirected layouts complicate the identification of the topology within the domain graph because such layout algorithms do not distinguish between different biological types of nodes or edges. In addition, protein nodes should be placed close to their domains.

Consequently, we developed the special layout algorithm RadialLayout, combining elements from radial and layered layout algorithms [29]. Protein and domain nodes are separated by placing them on two concentric circles around a common center such that no nodes overlap (Figure 1). Then a permutation of the nodes that minimizes the number of protein-domain edge crossings is determined by using bilayer crossing minimization techniques where each circle is interpreted as a layer of a bilayer drawing. Since the bilayer crossing minimization problem is NP-hard [30], we apply an adaption of the so-called barycenter heuristic [31] such that our approach can be applied to large networks of several hundred nodes. The barycenter heuristic keeps the permutation on one of the layers L1 fixed, computes for each node on the second layer L2 the barycenter with regard to be the position of its neighbors on L1, and sorts the nodes on L2 accordingly. This computation is continued with alternating roles of the two layers until no further improvement on the number of crossings is achieved.

Counting the edge crossings in each pass can be the bottleneck of this approach with regard to running time. Thus, we implemented an efficient bilayer cross counting method that proved to be very fast in experiments [32] and has $\mathrm{O}(|\mathrm{E}| \log \mid \mathrm{V}$ small $\mid)$ asymptotic running time where Vsmall is the smaller set of proteins and domains. Minimizing crossings between proteindomain edges also ensures that proteins can be placed close to their constituent domains, which is done in a positioning step after the crossing minimization. To separate not only the nodes, but also the edges in the domain graph, the domain-domain interaction edges are drawn through the center of the inner circle, while the protein-protein interaction edges are routed around the outer circle. The latter is done in clockwise or counterclockwise direction, using the shorter of the two possible routing paths. When integrating expression data, the placement of protein and domain nodes further takes the different node types into account (Section 2.4), and nodes of identical type are placed close to each other (Figure 2).

\subsection{Domain Graph Views}

We support three different network views (Figure 5) of domain graphs to adjust the visualization. Switching between the views is possible at any time. The most detailed view is the extended view in which the domain nodes are shown for each protein separately. However, if a protein contains several instances of the same domain, the domain is represented only once for this protein. The complete domain architecture of the proteins including domain repeats is displayed in the graphical representation (Figure 6).

The compact view reduces the number of nodes and edges in the domain graph by merging all domains of the same family into a single meta-node and by linking all proteins containing this domain to the meta-node. This compact view is especially useful if a large number of proteins contains domains of the same family. The third view is the protein interaction network view in 

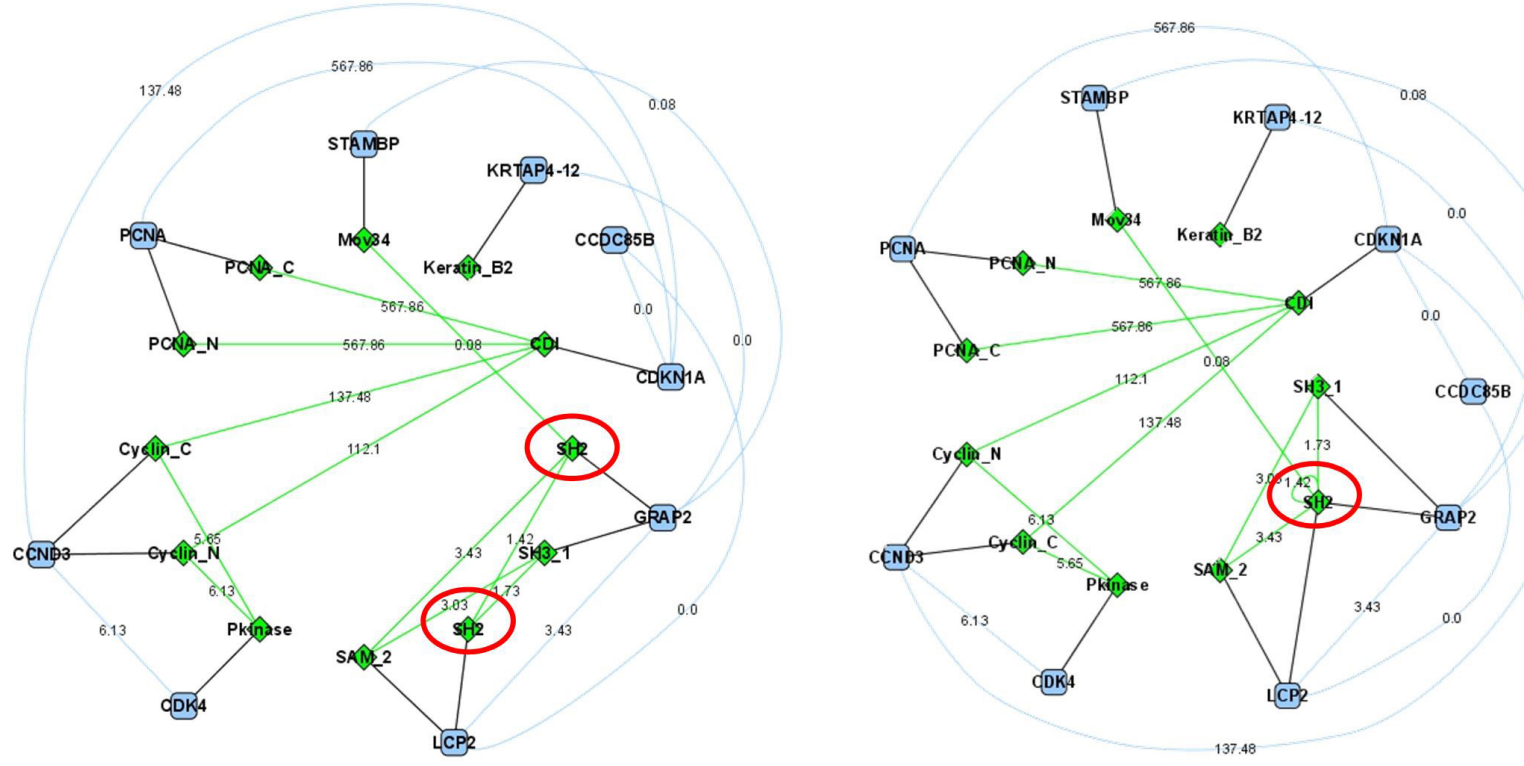

Figure 5: The left-hand side shows the extended view and the right-hand side the compact view of the same domain graph. The SH2 domains (in red circles) of the proteins LCP2 and GRAP2 are displayed separately in the extended view and are merged into a single meta-node in the compact view.

which only the protein interaction network is displayed. The user can then select the proteins of interest and add the underlying domain-domain interactions for these proteins. This view is useful for exploring the input protein interaction network in a step-by-step fashion without losing track of the relevant data.

\subsection{Additional Plugin Features}

The DomainGraph plugin provides additional graphical and textual information on the proteins and domains contained in the domain graph. The graphical information is displayed if the user double-clicks on a protein node. The domain architecture of the respective protein is shown together with the underlying exon structure, and, in case of Affymetrix expression data integration, the probesets annotated to a certain exon are included. All domains and exons in the graphics are linked to the respective source databases, and if the user clicks on a domain or exon, the relevant web page is opened in the user's web browser.

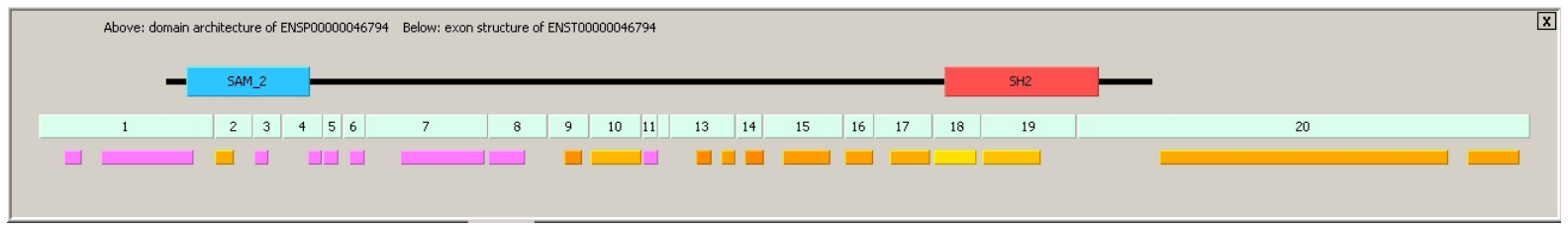

Figure 6: Graphical information on the LCP2 protein with integrated expression data. At the top, the domain architecture of the protein is depicted. In the center, the underlying exon structure is displayed, including the $3^{\prime}$ and 5' UTRs of the transcript sequence. At the bottom, the probesets annotated to the exons are shown. Probesets are colored according to their presence (color gradient from yellow to red) and absence (pink).

The probesets in the picture are colored according to the imported expression and p-value data. A color gradient for the expression strength is applied to all probesets that are present according 


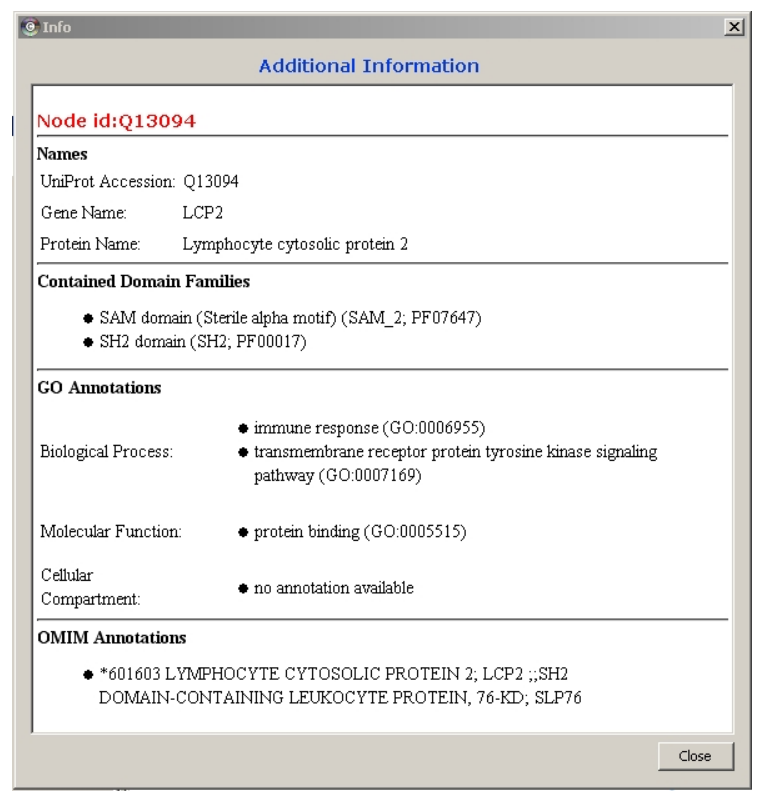

Figure 7: Textual information on the LCP2 protein, concerning the protein and constituent domains and the Gene Ontology (GO) and OMIM disease annotations.

to the p-value data, while absent probesets are uniformly colored pink. If the proteins in the interaction network are identified with UniProtKB accession numbers, they are mapped to the corresponding Ensembl proteins first because the Affymetrix mapping is provided for Ensembl transcript ids only. Figure 6 shows the graphical information provided for the lymphocyte cytosolic protein 2 (LCP2) that is contained in the sample domain graphs in Figures 1 and 2. As can be seen in Figure 2, the SAM_2 domain is regarded as spliced out according to the imported sample Affymetrix data since the majority of probesets covering this domain-coding region are absent (pink). However, all probesets covering the region of the $\mathrm{SH} 2$ domain show expression according to the imported p-value and expression data, and the $\mathrm{SH} 2$ domain is thus considered as expressed.

Tooltip information on all elements in the graphical representation is available if the user hovers the mouse over a domain, exon or probeset. For example, regarding the domains and exons, information on their lengths and on the start and end positions of a domain within an exon is available. The tooltips of the probesets display information like the length of the probeset, the expression strength, and the p-value.

The differentiation of present and absent probesets is computed according to a pre-defined threshold value, which is set to 0.05 by default. Default values are also defined for the required percentage of expressed probesets within domain-coding regions or the complete transcript sequence. However, the user has the possibility of adjusting all parameters in an options dialog provided by the plugin.

In addition to the graphical information, textual information like Gene Ontology and OMIM disease annotation is available for proteins and domains for obtaining a quick overview of the protein or domain of interest (Figure 7). The protein and domain nodes are linked to their source databases via a node context menu so that the user can easily retrieve all available external information. The user may also adjust a constructed domain graph in the plugin options. It is possible to display or hide domain nodes that do not form domain-domain interactions in the given domain graph. This option is especially useful for sparse graphs when many domain nodes can be hidden in order to focus on relevant information only. 


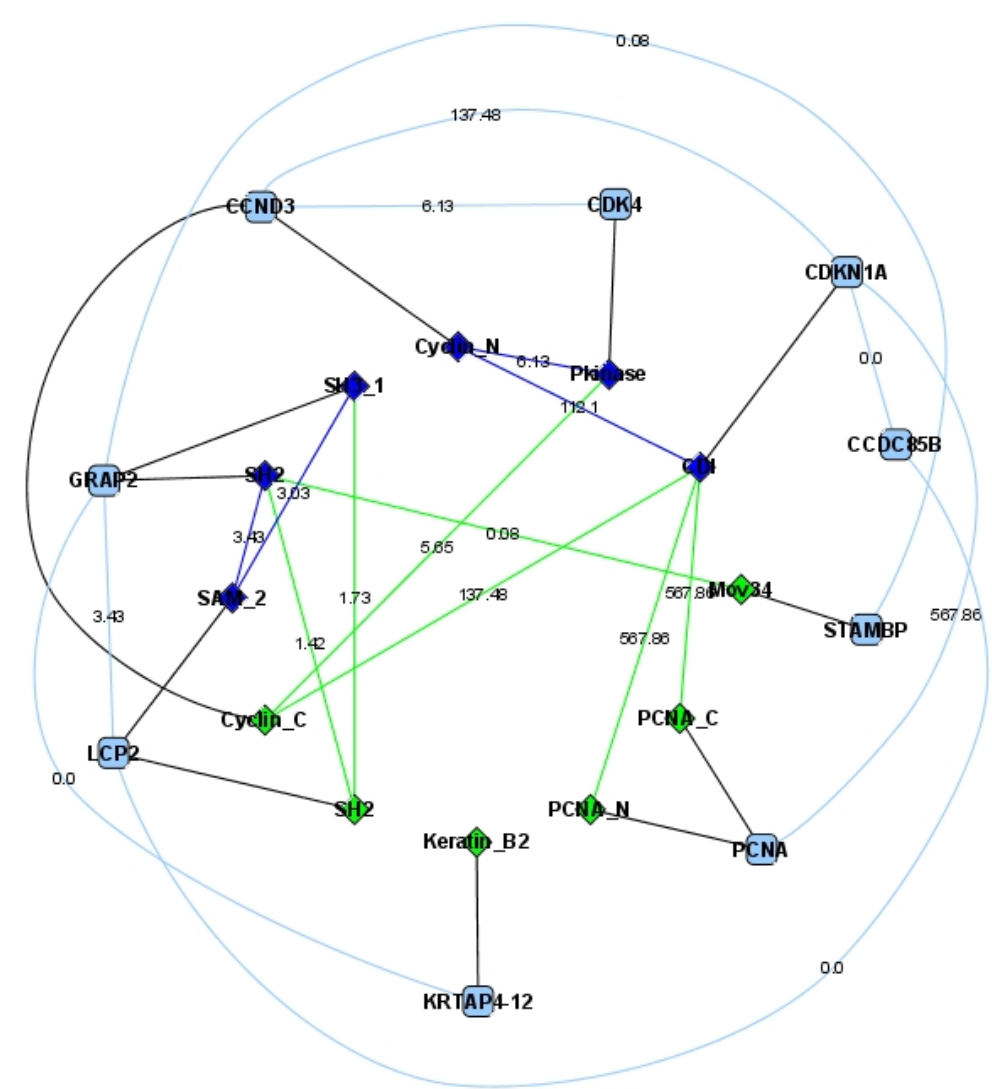

Figure 8: Intersection graph computed of the domain graphs in Figures 1 and 2. Nodes and edges contained in both domain graphs with different type annotation are colored dark blue.

If the domain graph is derived from a dataset of predicted domain interactions, confidence scores are provided with the protein and domain interaction edges. Interactions with a confidence score below a user-defined threshold can be discarded from the domain graph to focus on more meaningful interactions. Confidence scores of predicted domain interactions can be visualized by representing more confident interactions with wider edges.

\subsection{Visual Analysis Tools}

A possible application of the DomainGraph plugin is the detection of different alternative splicing patterns in various tissue samples or healthy and diseased samples. Such a comparison can be very challenging depending on the size of the constructed domain graph. To facilitate such an analysis, we implemented the computation of the intersection, union and difference of domain graphs. These analysis methods do not only take the nodes and edges into account, but also their types (e.g., an expressed domain or a spliced domain). For this purpose, we introduce a new node and edge type, the combination type (Section 2.4), which enables the user to directly identify expression differences between two domain graphs.

For example, if a domain is contained in both analyzed domain graphs but is normally expressed in one and spliced out in the other, this node will be especially highlighted in the resultant domain graph. Computing the intersection of the two domain graphs in Figures 1 and 2 gives the domain graph presented in Figure 8. All nodes and edges shared between the two graphs in Figures 1 and 2, but having different biological types, are highlighted in dark blue, pointing the user to potentially important differences. 


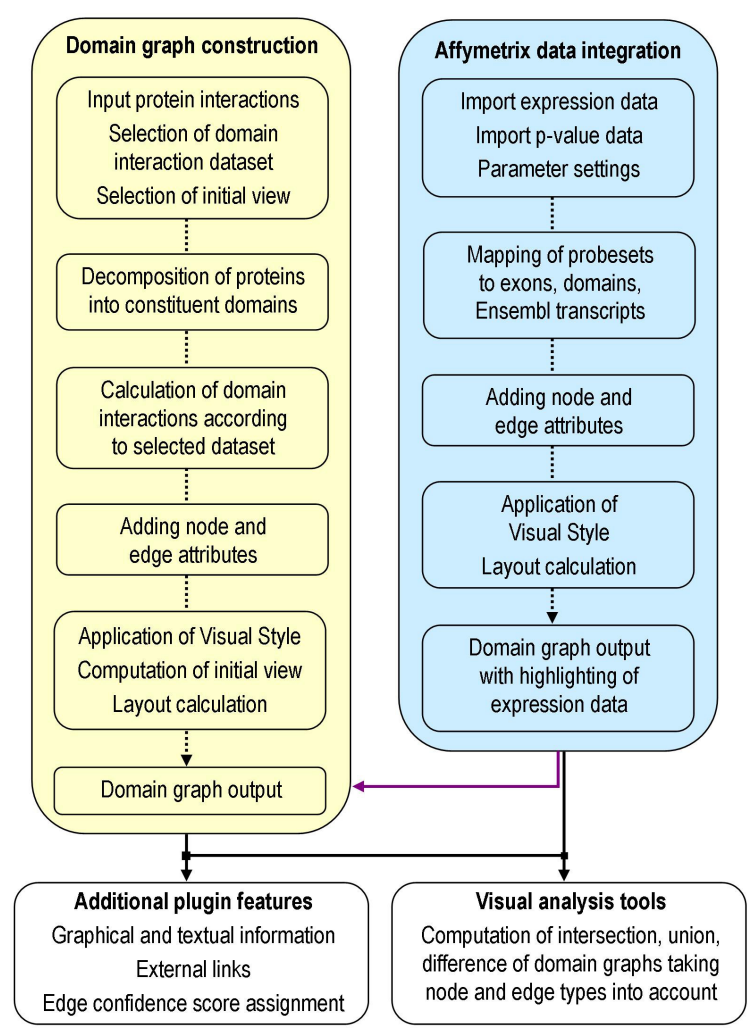

Figure 9: Schema of the domain graph construction and the Affymetrix expression data integration. The plugin features and analysis methods can both be applied to domain graphs with and without expression data as shown by the black arrows at the bottom. The purple line indicates that the second module can optionally be applied to a constructed domain graph.

\subsection{Implementation Overview}

The implementation of the Java plugin DomainGraph consists of two major modules (Figure 9). The first module handles the domain graph construction. The plugin queries the embedded database for the decomposition of proteins into their constituent domains and also for the calculation of occurring domain-domain interactions. Node and edge attributes are added, for instance, for the application of the Visual Style, for the layout calculation, and for providing fast access to additional features. The second module handles the import of Affymetrix expression data. The imported expression and p-value data are mapped to the corresponding exons and thus transcripts and domains. The identification of domains spliced out according to the expression data and of complete gene suppression is performed as follows:

- The default $\mathrm{p}$-value threshold for the presence/absence call of a probeset is set to 0.05 .

- The probesets are mapped to the corresponding exons. An exon is regarded as expressed if at least $50 \%$ of the assigned probesets are expressed.

- All probesets mapped to the respective protein are counted. A protein is regarded as expressed if at least $50 \%$ of the probesets are expressed.

- The exons are mapped to the domains in which they are contained. A domain is regarded as expressed if more than $75 \%$ of the exons that form the domain are expressed. Otherwise, the domain is regarded as spliced out if the protein itself is expressed. 
The parameters applied to the integrated Affymetrix expression data are set by default as described above. However, before integrating own expression data, the user can easily customize the default values in an options dialog provided by the DomainGraph plugin.

\subsection{Embedded Database}

The DomainGraph plugin provides all data that are required for the construction of a domain graph, for the integration of Affymetrix expression data, and for obtaining the graphical and textual information. To this end, the plugin includes an embedded Apache Derby database that is built in the user's Cytoscape directory the first time DomainGraph is started. Using an embedded database has the advantage that the user does not need to connect to an external database via an internet connection, but can run the plugin on a local machine. In addition, using this database, the construction of the domain graph and the integration of Affymetrix data into the graph is faster than using an external database connection. Another important point is that the user has the possibility to import confidential or unpublished protein or exon expression data for visualization with the plugin.

The embedded database contains UniProtKB accessions numbers for the twelve supported species together with the decomposition into their constituent Pfam domains, which is obtained directly from UniProtKB release 12.8. For human proteins, a mapping between the UniProtKB accessions and Ensembl transcript and peptide identifiers from Ensembl release 47 is included. For each Ensembl transcript, the underlying exon structure is stored as well as the mapping of exons to Affymetrix probesets. The mapping between Ensembl transcript ids and probesets was obtained directly from Affymetrix, and the genomic coordinates of the probesets were then mapped to the corresponding Ensembl exons. Additionally, the database contains the Gene Ontology annotations for the proteins and domains and the OMIM disease annotation for the proteins. This information was taken from the UniProtKB release 12.8.

\section{Conclusions}

The DomainGraph plugin is a powerful tool for the integrative visual analysis of the effects of alternative splicing events on protein and domain interaction networks using imported Affymetrix Human Exon Array data. Loss of protein and domain interactions due to alternative splicing within domain-coding regions is specifically highlighted in the domain graph and thus can easily be identified by the user. The graphical representation of the proteins and domains together with the exon structures and probeset annotations illustrates the precise sequence positions of splicing events. Since the user can change all default parameters used in the process of the Affymetrix data integration, the plugin offers great customizability.

Moreover, additional information like GO and OMIM annotations for proteins and domains may direct the user to the impact of splicing events on cellular processes and to protein functions disturbed in disease. Using the novel analysis methods provided by the DomainGraph plugin, it also becomes possible to efficiently compare and discover variations of splicing patterns between different tissues and to find important differences between healthy and diseased tissues.

The future development of the DomainGraph plugin may include the visualization of protein complexes in addition to pairwise protein-protein interactions. To this end, new methods are 
required that derive and visualize all domain interactions occurring in protein complexes. Apart from Affymetrix Human Exon Array data, the import and incorporation of expression data produced for other organisms like mouse and rat or with microarrays by other vendors like Agilent or Illumina could be implemented.

\section{Acknowledgements}

Part of this work has been financially supported by the German National Genome Research Network (NGFN) and by the German Research Foundation (DFG), contract number KFO 129/12. The research has been conducted in the context of the BioSapiens Network of Excellence funded by the European Commission (grant number LSHG-CT-2003-503265).

\section{References}

[1] F. Ramírez, A. Schlicker, Y. Assenov, T. Lengauer, and M. Albrecht. Computational analysis of human protein interaction networks. Proteomics, 7(15):2541-2552, 2007.

[2] A. J. Matlin, F. Clark, and C. W. Smith. Understanding alternative splicing: towards a cellular code. Nature Reviews Molecular Cell Biology, 6(5):386-398, 2005.

[3] B. J. Blencowe. Alternative splicing: new insights from global analyses. Cell, 126(1):3747, 2006.

[4] A. Resch, Y. Xing, B. Modrek, M. Gorlick, R. Riley, and C. Lee. Assessing the impact of alternative splicing on domain interactions in the human proteome. Journal of Proteome Research, 3(1):76-83, 2004.

[5] A. E. Loraine, G. A. Helt, M. S. Cline, and M. A. Siani-Rose. Protein-based analysis of alternative splicing in the human genome. Proceedings of the IEEE Computer Society Bioinformatics Conference, 1:118-124, 2002.

[6] Affymetrix. GeneChip exon array system for human, mouse, and rat, 2005. http://www.affymetrix.com/support/technical/datasheets/ exon_arraydesign_datasheet.pdf.

[7] T. A. Clark, A. C. Schweitzer, T. X. Chen, M. K. Staples, G. Lu, H. Wang, A. Williams, and J. E. Blume. Discovery of tissue-specific exons using comprehensive human exon microarrays. Genome Biology, 8(4):R64, 2007.

[8] J. P. Orengo and T. A. Cooper. Alternative Splicing in the Postgenomic Era, volume 623 of Advances in Experimental Medicine and Biology, chapter Alternative splicing in disease, pages 212-223. Springer, 2007.

[9] P. J. Gardina, Tyson A. Clark, Brian Shimada, Michelle K. Staples, Qing Yang, James Veitch, Anthony Schweitzer, Tarif Awad, Charles Sugnet, Suzanne Dee, Christopher Davies, Alan Williams, and Yaron Turpaz. Alternative splicing and differential gene expression in colon cancer detected by a whole genome exon array. BMC Genomics, 7:325, 2006. 
[10] M. S. Cline et al. Integration of biological networks and gene expression data using Cytoscape. Nature Protocols, 2(10):2366-2382, 2007.

[11] A. Schlicker, C. Huthmacher, F. Ramírez, T. Lengauer, and M. Albrecht. Functional evaluation of domain-domain interactions and human protein interaction networks. Bioinformatics, 23(7):859-865, 2007.

[12] M. Albrecht, C. Huthmacher, S.C. Tosatto, and T. Lengauer. Decomposing protein networks into domain-domain interactions. Bioinformatics, 21(Suppl 2):ii220-ii221, 2005.

[13] E. Boutet, D. Lieberherr, M. Tognolli, M. Schneider, and A. Bairoch. UniProtKB/SwissProt: the manually annotated section of the UniProt KnowledgeBase. Methods in Molecular Biology, 406:89-112, 2007.

[14] P. Flicek, B. L. Aken, K. Beal, B. Ballester, M. Caccamo, Y. Chen, L. Clarke, G. Coates, F. Cunningham, T. Cutts, T. Down, S. C. Dyer, T. Eyre, S. Fitzgerald, J. FernandezBanet, S. Graf, S. Haider, M. Hammond, R. Holland, K. L. Howe, K. Howe, N. Johnson, A. Jenkinson, A. Kahari, D. Keefe, F. Kokocinski, E. Kulesha, D. Lawson, I. Longden, K. Megy, P. Meidl, B. Overduin, A. Parker, B. Pritchard, A. Prlic, S. Rice, D. Rios, M. Schuster, I. Sealy, G. Slater, D. Smedley, G. Spudich, S. Trevanion, A. J. Vilella, J. Vogel, S. White, M. Wood, E. Birney, T. Cox, V. Curwen, R. Durbin, X. M. FernandezSuarez, J. Herrero, T. J. P. Hubbard, A. Kasprzyk, G. Proctor, J. Smith, A. Ureta-Vidal, and S. Searle. Ensembl 2008. Nucleic Acids Research, 36(Database issue):D707-714, 2008.

[15] R. D. Finn, John Tate, Jaina Mistry, Penny C. Coggill, Stephen John Sammut, HansRudolf Hotz, Goran Ceric, Kristoffer Forslund, Sean R. Eddy, Erik L. L. Sonnhammer, and Alex Bateman. The Pfam protein families database. Nucleic Acids Research, 36(Database issue):D281-288, 2008.

[16] R. D. Finn, M. Marshall, and A. Bateman. iPfam: visualization of protein-protein interactions in PDB at domain and amino acid resolutions. Bioinformatics, 21(3):410-412, 2005.

[17] A. Stein, R.B. Russell, and P. Aloy. 3did: interacting protein domains of known threedimensional structure. Nucleic Acids Research, 33(Database issue):D413-417, 2005.

[18] S. K. Ng, Z. Zhang, S. H. Tan, and K. Lin. InterDom: a database of putative interacting protein domains for validating predicted protein interactions and complexes. Nucleic Acids Research, 31(1):251-254, 2003.

[19] Y. Liu, N. Liu, and H. Zhao. Inferring protein-protein interactions through highthroughput interaction data from diverse organisms. Bioinformatics, 21(15):3279-3285, 2005.

[20] R. Riley, C. Lee, C. Sabatti, and D. Eisenberg. Inferring protein domain interactions from databases of interacting proteins. Genome Biology, 6(10):R89, 2005.

[21] P. Pagel, Matthias Oesterheld, Oksana Tovstukhina, Norman Strack, Volker Stumpflen, and Dmitrij Frishman. DIMA 2.0 - predicted and known domain interactions. Nucleic Acids Research, 36(Database issue):D651-655, 2008. 
[22] H. Lee, M. Deng, F. Sun, and T. Chen. An integrated approach to the prediction of domain-domain interactions. BMC Bioinformatics, 7:269, 2006.

[23] X. W. Chen and M. Liu. Prediction of protein-protein interactions using random decision forest framework. Bioinformatics, 21(24):4394-4400, 2005.

[24] R. Jothi, P. F. Cherukuri, A. Tasneem, and T. M. Przytycka. Co-evolutionary analysis of domains in interacting proteins reveals insights into domain-domain interactions mediating protein-protein interactions. Journal of Molecular Biology, 362(4):861-875, 2006.

[25] R. S. Wang, Y. Wang, L. Y. Wu, X. S. Zhang, and L. Chen. Analysis on multi-domain cooperation for predicting protein-protein interactions. BMC Bioinformatics, 8:391, 2007.

[26] K. S. Guimaraes, R. Jothi, E. Zotenko, and T. M. Przytycka. Predicting domain-domain interactions using a parsimony approach. Genome Biology, 7(11):R104, 2006.

[27] J.-F. Rual et al. Towards a proteome-scale map of the human protein-protein interaction network. Nature, 437(7062):1173-1178, 2005.

[28] Affymetrix. Affymetrix Power Tools (APT), 2008. http://www.affymetrix.com/ support/developer/powertools/index . affx.

[29] D. Emig, K. Klein, A. Kunert, P. Mutzel, and M. Albrecht. Visualizing domain interaction networks and the impact of alternative splicing events. In Proceedings of the 12th International Conference on Information Visualization (IV08), Washington, DC, USA, 2008. IEEE Computer Society.

[30] M. Garey and D. Johnson. Crossing number is NP-complete. SIAM Journal on Algebraic and Discrete Methods, 4:312-316, 1983.

[31] W. Barth, M. Jünger, and P. Mutzel. Simple and efficient bilayer cross counting. Journal of Graph Algorithms and Applications, 8(2):179-194, 2004.

[32] K. Sugiyama, S. Tagawa, and M. Toda. Methods for visual understanding of hierarchical systems. IEEE Transactions on Systems, Man and Cybernetics, 11(2):109-125, 1981. 